\title{
Establish and Apply a Safe and Convenient Pseudovirus-Based Assay to Detect Neutralizing Antibodies against Influenza A (H7N9) Virus
}

\author{
Li Zhang *, Jing Lu, Jinxing Li, Rong Tang, Haiyan Peng, Fanyue Meng and \\ Yuemei Hu
}

Jiangsu Provincial Center for Disease Prevention and Control, Nanjing 210009, China; hhtt319@163.com (J.L.); ingxin42102209@126.com (J.L.); tangrongtr@126.com (R.T.); sun_tutu@126.com (H.P.); mfy19780712@163.com (F.M.); huyuemei@hotmail.com (Y.H.)

*Correspondence: zhangli411@yeah.net; Tel.: +86-25-8375-9418

\begin{abstract}
In March 2013, a novel avian influenza A H7N9 virus was emerged in China, which cause rapidly progressive pneumonia and with a high fatality rate. Serologic studies to evaluate neutralizing antibodies of infected patients and birds are invaluable tools for immunogenicity research of H7N9 and epidemiological investigation. Conventional neutralization assays are laborious and time-consuming which also hampered by biosafety requirement. In this study, We construct and produce pseudovirus bearing the full-length hemagglutinin (HA) of H7N9 virus in the Env-defective, luciferase-expressing HIV-1 backbone. The production of lentiviral pseudovirus was analysed by HA gene specific real-time reverse-transcription PCR, transmission electron microscopy (TEM), and Western Blot assay to prove the nucleic acid replication, the morphology of virus, and the expression of HA protein in pseudovirus. After that pseudovirus based inhibition assay was established to detect neutralizing antibodies of a panel of serum samples. Our results demonstrated that H7N9 pseudovirus which had single-cycle infection was generated. By comparing the neutralization antibody titers, pseudovirus based neutralization test could be recognized as an alternative of conventional microneutralization (MN). Hence, we conclude that it is possible to use pseudovirus inhibition assay to screen sera samples, as well as evaluate vaccine-induced neutralizing antibodies against H7N9 virus.
\end{abstract}

Keywords: H7N9 avian influenza; pseudovirus; neutralization assay; relative luminescence units (RLU) 


\section{Introduction}

Since March 2013 an outbreak caused by the avian-originated influenza A virus H7N9 subtype was emerged in eastern China[1]. Patients were characterized by rapidly progressive pneumonia, respiratory failure, acute respiratory distress syndrome and even with $35 \%$ fatality rate [2-4]. It has caused serious concerns for public health throughout the world.

Influenza A virus (IAV) are enveloped RNA viruses which belongs to the family Orthomyxoviridate. Its genome consists of eight negative-sense RNA segments that together code for a minimum 10 proteins. Classification of IAV is based on the two surface proteins hemagglutinin (HA) and neuraminidase (NA). To date, 16 HA (H1H16) and 9 NA (N1-N9) subtypes have been identified in avian natural reservoirs[5].This novel avian influenza A H7N9 virus originated from re-assortment of at least four avian influenza viruses. The $\mathrm{H} 7$ gene is derived from avian influenza viruses of duck origin, and N9 gene transferred from migratory birds infected with avian influenza viruses. Other six gene segments are from two different groups of avian $\mathrm{H} 9 \mathrm{~N} 2$ viruses. After re-assortment, the novel H7N9 virus has acquired some characteristic mutations that facilitate viral recognition of human-type receptors and efficient replication in mammals. [6, 7]

The real-time reverse-transcription PCR (RT-PCR) is a recommended method to detect the shedding of viral RNA which is often performed in early diagnosis [8,9]. After the virus shedding period, serologic assays are performed to detect virus specific antibodies $[10,11]$.

The haemagglutination-inhibition (HAI) assay is a traditional, reliable and relatively simple method for serological screening of influenza virus [12] even through it is well know that HI titers can vary between laboratories. As an alternative, HA subtypes specific enzyme-linked immunosorbent assays (ELISA) are used to detect specific antibodies for HA [13]. Both these two assays cannot detect virusspecific neutralizing antibodies. Virus neutralization assays, which are represented by microneutralization (MN), aims to evaluate neutralizing antibodies. Moreover, $\mathrm{MN}$ is limited for the need of biosafety-level 3 (BSL-3) containment facilities [14], and the process is time-consuming and laborious. 
Lentivirus mediate pseudovirus bearing heterologous viral glycoproteins have been described for several avian influenza viruses including H7N7 [15], H5N1[16, 17],H3N2[18] and H1N1[19]. Pseudovirus particles have the ability of a single round infection and do not produce live virus. Therefore it can be produced in lower biosafety requirements than the wild type virus. Pseudovirus based assay for detecting subtype H7N9 neutralizing antibodies have been recognized as reliable and safe alternatives for $\mathrm{MN}$.

Here we describe a system to produce lentiviral pseudotype particles with $\mathrm{H} 7$ hemagglutinin isolated from a patient in $\mathrm{WuXi}$ in 2013. H7N9 pseudovirus shows similar characteristics as live virus. Our data indicate that this pseudovirus will be a useful tool in serodiagnostic assay, evaluation of neutralizing antibodies, identification of drug-resistant mutations.

\section{Results}

\subsection{Production of infectious lentiviral H7N9 pseudovirus.}

To obtain pseudoviruses bearing HA segment of H7N9, full-length of HA genes were constructed to pcDNA3.0 vector to form pcDNA3.0-HA recombinant plasmid. $293 \mathrm{~T}$ cells cultured in dish were co-transfected with lentivirus vector pCD-HR'8.3, pCD-HR-Luc and pcDNA3.0-HA by using lipofectamine 2000. 48h after transfection, supernatant was clarified by centrifugation and stored at $-80^{\circ} \mathrm{C}$ in aliquots.

RNA of supernatant was extracted and RT-PCR was performed to test the amplification of HA segments. By using specific primers and probes, HA genes in pseudovirus were amplified and the average $\mathrm{Ct}$ value was 14.3. It suggested that after co-transfection the mRNA of pseudovirus carrying HA gene was transcribed and translated in host cells.

To further understand the morphology of pseudovirus, the supernatant of transfected $293 \mathrm{~T}$ cells was characterized by electron microscopy. As shown in Figure 1, the morphology of pseudovirus resembles the morphology of influenza virus with spikes on the surface, and the size ranged from approximately 100 to $120 \mathrm{~nm}$.

To confirm the HA expression in pseudovirus particles, Western Blot was performed. As shown in Figure 2, the band with sizes of $75 \mathrm{kD}$ corresponding to the 
size of HA protein was revealed by using H7N9 virus immunized mouse sera. It demonstrated that HA protein had been expressed in lentiviral particles successfully.

\subsection{Infectivity kinetic pattern and CCID50 detection of pseudovirus}

As described in former, pseudovirus particles bearing HA segment of H7N9 were generated. Pseudovirus particles have the ability of a single round infection and do not produce live virus. As the luciferase reporter gene was carried in the genomic RNA of the pseudovirus, its infectivity can be quantified by luciferase activity in virusinfected cells. The kinetic pattern of H7N9 pseudovirus was determined by measuring luciferase activity of infected MDCK cells over a time course. The value of RLU peaked at 16 hours post-infection which about $10^{6} \mathrm{RLU} / \mathrm{mL}$ and decreased gradually with time (Figure 3).

For CCID50 detection, MDCK cells were infected by serially diluted pesudovirus. 16 hours post-infection, which was the peak point of RLU, cells were harvested and RLU was tested. By using the Reed-Muench method, the $50 \%$ cell culture infective dose was $10^{3.88}$.

\subsection{Pseudovirus particle neutralization test}

The Pseudovirus particle based neutralization assay was carried out on 8 human serum and 8 mouse serum samples. Neutralizing antibodies of these samples were also detected by microneutralization assay using live virus. Eight convalescent-phase serum samples confirmed by clinical diagnosis and real-time RT-PCR were all able to inhibit the single infection of pseudovirus by reducing RLU values of luciferase reporter genes (Fig 4A). Pseudovirus neutralization had no species-specificity, the same results were also found in mouse samples (Fig 4B). Comparing results of neutralizing antibody titers, these two methods had a good correlation, and no statistically significant difference was found both in human and mouse serum samples. 


\section{Discussion}

Pseudovirus has the ability to express heterologous protein in the surface of particles. As live virus, it could undergo the steps of attachment, fusion and expression of reporter gene, but it cannot form infectious virus due to lacking of structural genes [20]. In this study we demonstrated that H7N9 HA pseudovirus exhibited similar characters as wild type H7N9 live viruses in replication, morphology and HA expression on the surface of particles.

We have confirmed that H7N9 HA pseudovirus based neutralization assay is an alternative approach for serologic screening when H7N9 emergent in China. Results of pseudovirus neutralization assay have no difference in antibody titers with conventional microneutralization. Besides, pseudovirus neutralization assay is more convenient and time-saving than the former. The process of pseudovirus package and neutralizing antibodies test could be completed about 2 weeks. However, traditional virus isolation, TCID50 detection and neutralization may need several months even years.

Pseudovirus based inhibition could be used in high pathogenicity virus in a lower BSL laboratories. The propagation and operation of avian H7N9 virus need BSL-3 containment facilities, but pseudovirus related assay can be performed in laboratories equipped with BSL-2 facilities. And it could be used for some viruses which are hard to culture or isolate or even impossible to get live virus [21, 22], as genes of target virus could be downloaded and synthesized for psedovirus package

In this study, RLU values were used as the endpoint of H7N9 neutralization assay. RLU values were measured by using Fluorometer, which is suited for automated high throughput screening in 96-well even 384-well plates. So it is convenient to get largescale seroprevalence studies on human and animal sera.

Therefore, in this manuscript, the pseudovirus of provides a safe, time-saving, quantitative, and high-throughput tool for many basic researches of H7N9 avian virus viruses. 


\section{Materials and methods}

\subsection{Cells, viruses and sera}

293 T cells and Madin-Daby canine kidney (MDCK) cells were both cultured in Dulbecco's Modified Eagle Medium (DMEM; Gibco) supplemented with 10\% fetal bovine serum (FBS), penicillin $(100 \mathrm{U} / \mathrm{ml})$ and streptomycin $(100 \mu \mathrm{g} / \mathrm{ml})$ at $37^{\circ} \mathrm{C}$ with 5\% $\mathrm{CO}_{2}$. Avian influenza H7N9 viruses A/Wuxi/1/2013 (H7N9) (GenBank: KF034914.1) which was isolated by our laboratory was grown in MDCK cells and used for cDNA in this study. BALB/c mice were immunized with inactived H7N9 virus and sera were collected in the former paper [23]. Human sera $(n=8)$ were collected at convalescent-phase of H7N9 infected patients confirmed by viral RNA detection using real-time polymerase chain reaction kit ( World Health Organization TaqMan assay) provided by WHO Collaborating Center for Reference and Research on Influenza at Chinese National Influenza Center. Negative human sera were collected and kept by our laboratory.

\subsection{Recombinant plasmid construction}

The QIAamp Viral RNA kit (Qiagen, Santa Clarita, CA, USA) was used to extract genomic RNA of H7N9 virus from supernatant of infected MDCK cells. The extracted RNA was subjected to reverse transcription PCR (RT-PCR) using Transcriptor High Fidelity cDNA Synthesis Kit (Roche, Penzberg, Germany) to generate cDNA. The HA gene was amplified with primers containing Kpn I and Xho I enzyme sites: F-HA, 5'- GGGGTACC CACCATGAACACTCAAATCCTG-3' and R-HA 5' CCGCTCGAGTTATATACAAATAGTGCACC-3'. The PCR segments were digested with specific enzymes and cloned to pcDNA3.0 vector to form pcDNA3.0-HA recombinant plasmid. 


\subsection{Production of H7N9 pseudovirus bearing HA}

To generate H7N9 pseudovirus, we carried the procedure as ViraPower ${ }^{\mathrm{TM}}$

Lentiviral Expression Systems kit (Invitrogen, USA). Briefly, 6×106 293T cells

cultured in a $10 \mathrm{~cm}$ dish were co-transfected with $3 \mu \mathrm{g}$ pcDNA3.0-HA and $3 \mu \mathrm{g}$ pCD-

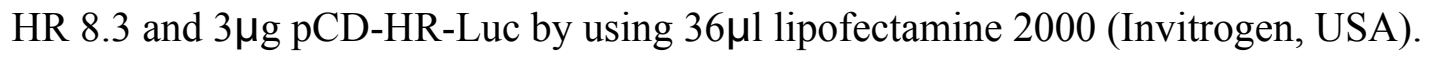

Cells were washed once with phosphate-buffered saline and the medium was replaced with DMEM (10\% FCS) at $6 \mathrm{~h}$ post-transfection. The pseudovirus containing

supernatant was harvested at $48 \mathrm{~h}$ and stored at $-80^{\circ} \mathrm{C}$ in aliquots until used in the later assay.

\subsection{Reverse transcription-PCR (RT-PCR)}

Supernantants from the co-transfected 293T cells were collected and RNA was extracted using QIAamp Viral RNA Mini Kit (Qiagen, Germany) according to the manufacturer's instructions. RT-PCR was performed using the SuperScript III Platinum One-Step Quantitative RT-PCR kit (Invitrogen, USA) on an ABI 7900 real-time PCR system (ABI, USA) with WHO issued primers and probes according to the WHO recommended protocol.

\subsection{Transmission electron microscopy (TEM)}

To perform electron microscopy, 48 hours post-transfection, 293T cells were fixed with $0.25 \%$ glutaraldehyde and $1 \%$ osmium tetraoxide, dehydrated with ethanol, and then embedded in epon resin. Thin sections were stained with lead citrate and uranyl acetate and observed by electron microscope.

\subsection{Western Blot Analysis}

Culture supernatants were harvested without concentration, then electrophoresed in NuPAGE 4\%-12\% Bis-Tris gradient gel and transferred to nitrocellulose membrane 
(GE Healthcare, Piscataway, USA). Membranes were blocked by 10\% skimmed milk for $1 \mathrm{~h}$ at room temperature (RT), and incubated with diluted (1:1000) H7N9 virus immunized mouse sera for another hour at RT. After intensive washing, the membranes were incubated with HRP- conjugated second antibodies. Protein bands were visualized by 3,3'diaminobenzidine (DAB) (Boster Bio, Wuhan, China) according to the manufacturer's instructions.

\subsection{Determination of CCID50}

A tube of pseudovirus was taken out of the $-80^{\circ} \mathrm{C}$ refrigerator and thawing in room temperature for use. MDCK cells were infected by adding pseudovirus, and the infectivity of pseudovirus was reflected by testing the relative luciferase units (RLU ) of cell lysis. After infection, the value of RLU was tested at an interval of 4 hours. The titration of pseudovirus particles was determined by measuring the $50 \%$ cell culture infective dose (CCID50) using a microtitration assay. Briefly, 100 $\mu$ l of pseudovirus supernatants were diluted serially 5-fold in quadruplicate in 96-well plates. About $2 \times 10^{4}$ MDCK cells were added and mixed gently to each well. After incubation at $37^{\circ} \mathrm{C}$ with $5 \% \mathrm{CO}_{2}$ for $16 \mathrm{~h}$, cells were harvested for measuring luciferase activity using a luciferase assay kit (Promega, Madison,WI,USA) according to the manufacturer's instructions. Briefly, cells were lysed by adding $100 \mu \mathrm{l}$ of reporter lysis buffer (Promega), and the RLU in cell lysates were measured using Fluorometer. The RLU value which exceeds the mean +2 standard deviation (S.D.) of negative control was considered positive. The CCID50 which was determined as the median (50\%) tissue culture infective dose could be calculated by the ReedMuench method. 


\subsection{Pseudovirus particle neutralization test}

Each human sample need to be heat-inactivated at $56^{\circ} \mathrm{C}$ for $30 \mathrm{~min} .50 \mu \mathrm{l}$ of serum was diluted serially 2-fold in triplicate from 1:8 to 1:1024 in 96-well plates. H7N9 pseudovirus (200 CCID50 per 50 $\mu$ l) were added to each well to the plate and incubated at $37^{\circ} \mathrm{C}$ with $5 \% \mathrm{CO}_{2}$ for $1 \mathrm{~h}$. After the incubation, MDCK cells $\left(2 \times 10^{4}\right.$ cells in $100 \mu \mathrm{l}$ ) were added to each well and incubation at $37^{\circ} \mathrm{C}$ with $5 \% \mathrm{CO}_{2}$ for $16 \mathrm{~h}$. The RLU values of the infected cells were measured at the end time. The 50\% inhibitory concentration (IC50) titer was determined by the reciprocal of the last dilution that resulted in $\geq 50 \%$ reduction of luciferase activity. The inhibition percentage was calculated as follows:

$$
\text { Inhinition percentage }=\frac{\text { RLA in virus challenge control }- \text { RLA in serum inhibition well }}{\text { RLA in virus challenge control }} \times 100 \%
$$

\subsection{Statistical Analysis}

Statistical significance of the serum neutralizing antibody titer datum was assessed using the one-way ANOVA and Tukey HSD test. A p-value $<0.05$ was considered significant. Statistical analyses were done using GraphPad Prism 5.0 software.

\section{Author Contributions}

Li Zhang, Jing Lu, Fanyue Meng and Yuemei Hu conceived and designed the experiments. Li Zhang, Jinxing Li, Haiyan Peng and Rong Tang conducted the experiments. Li Zhang, Jing Lu analyzed the data. Li Zhang and Jing Lu wrote the manuscript.

\section{Conflicts of Interest}

The authors declare no conflict of interest. 


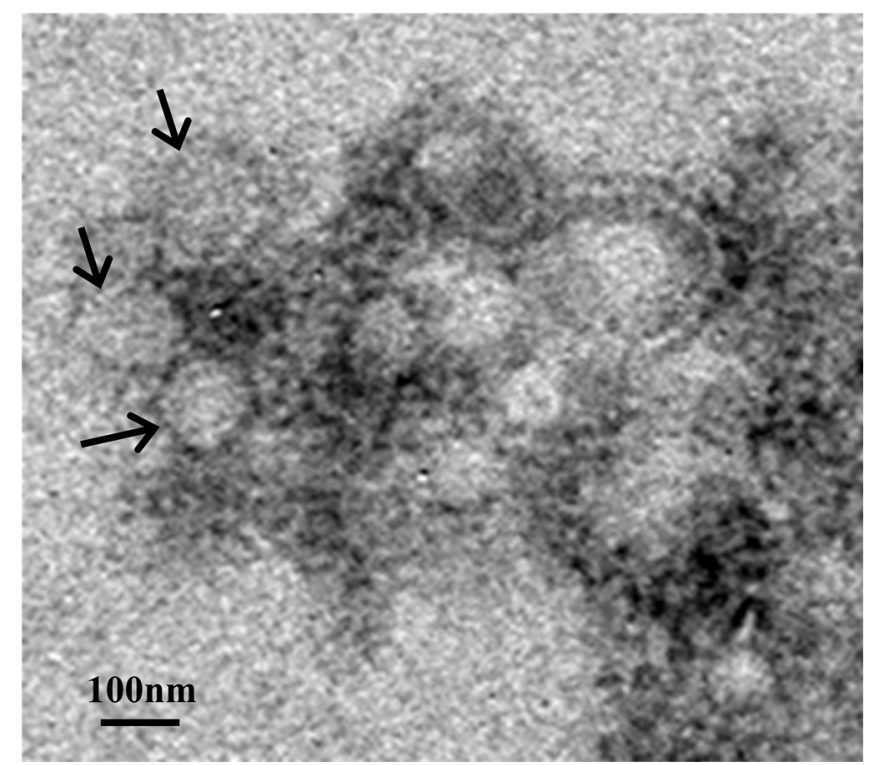

Fig. 1. Electron microscopy image of pseudovirus particles. Transfected $293 \mathrm{~T}$ cells were fixed, dehydrated, embedded, stained with lead citrate and uranyl acetate, and observed by electron microscopy.

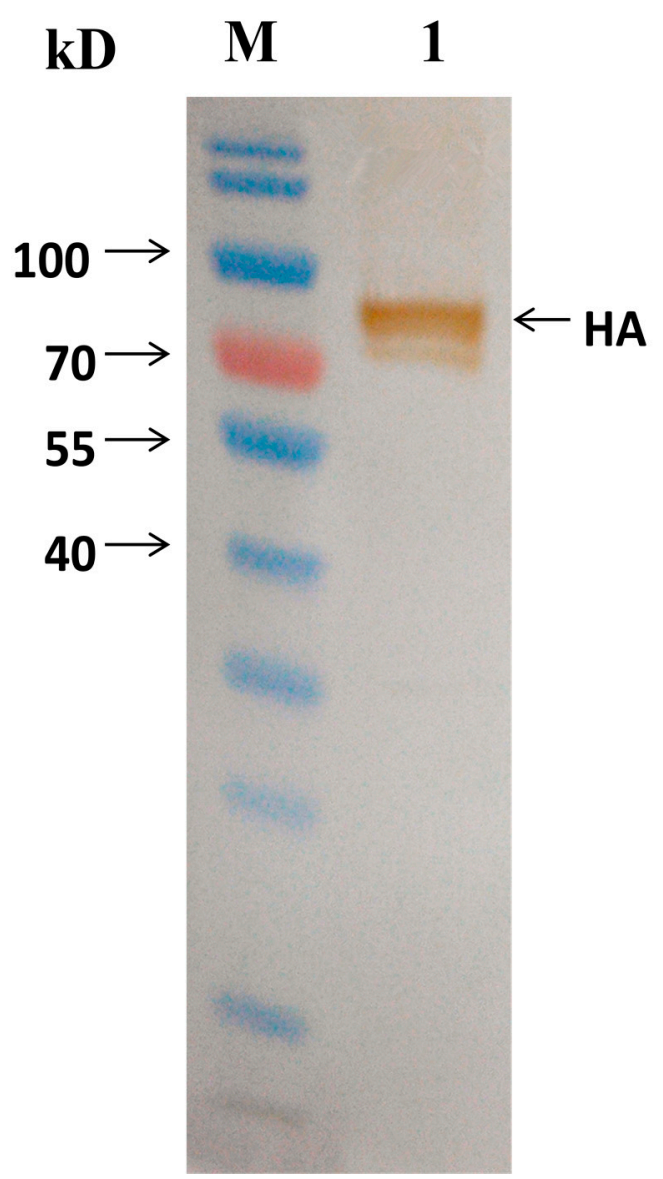

Fig. 2. Analysis of HA expressed in pseudovirus by Western blotting using H7N9 virus immunized mouse sera. The band with the molecular weight of $75 \mathrm{kD}$ is equal to the size of hemagglutinin (HA). 


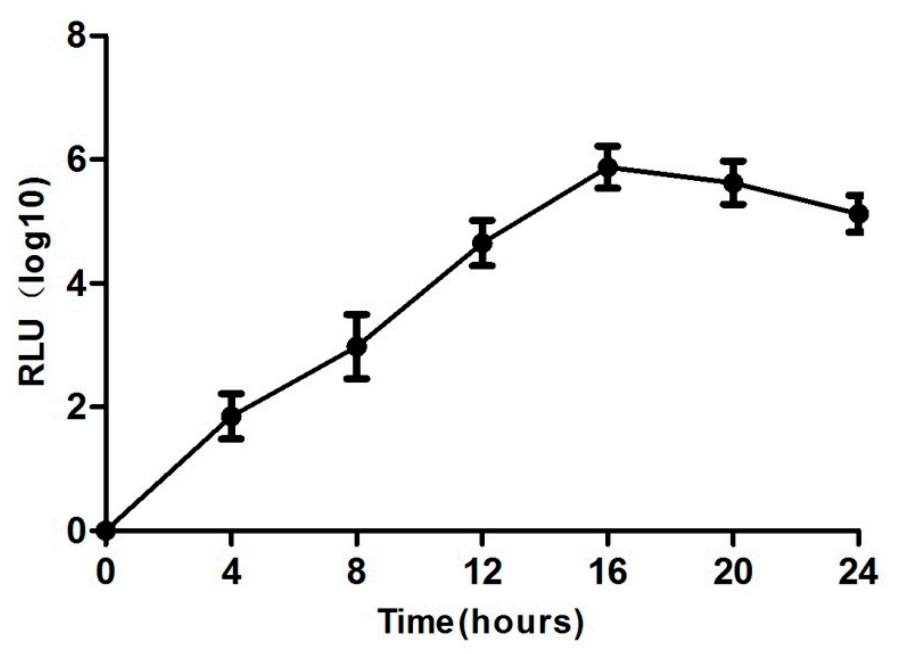

Fig. 3. To detect the kinetic pattern of pseudovirus infection processes. Pseudovirus was added to cells in 96-well plates. The RLU values were tested at an interval of 4 hours in 24 hour. Each performed in quadruplicate. Data was expressed as mean with a standard deviation (SD) bar. 

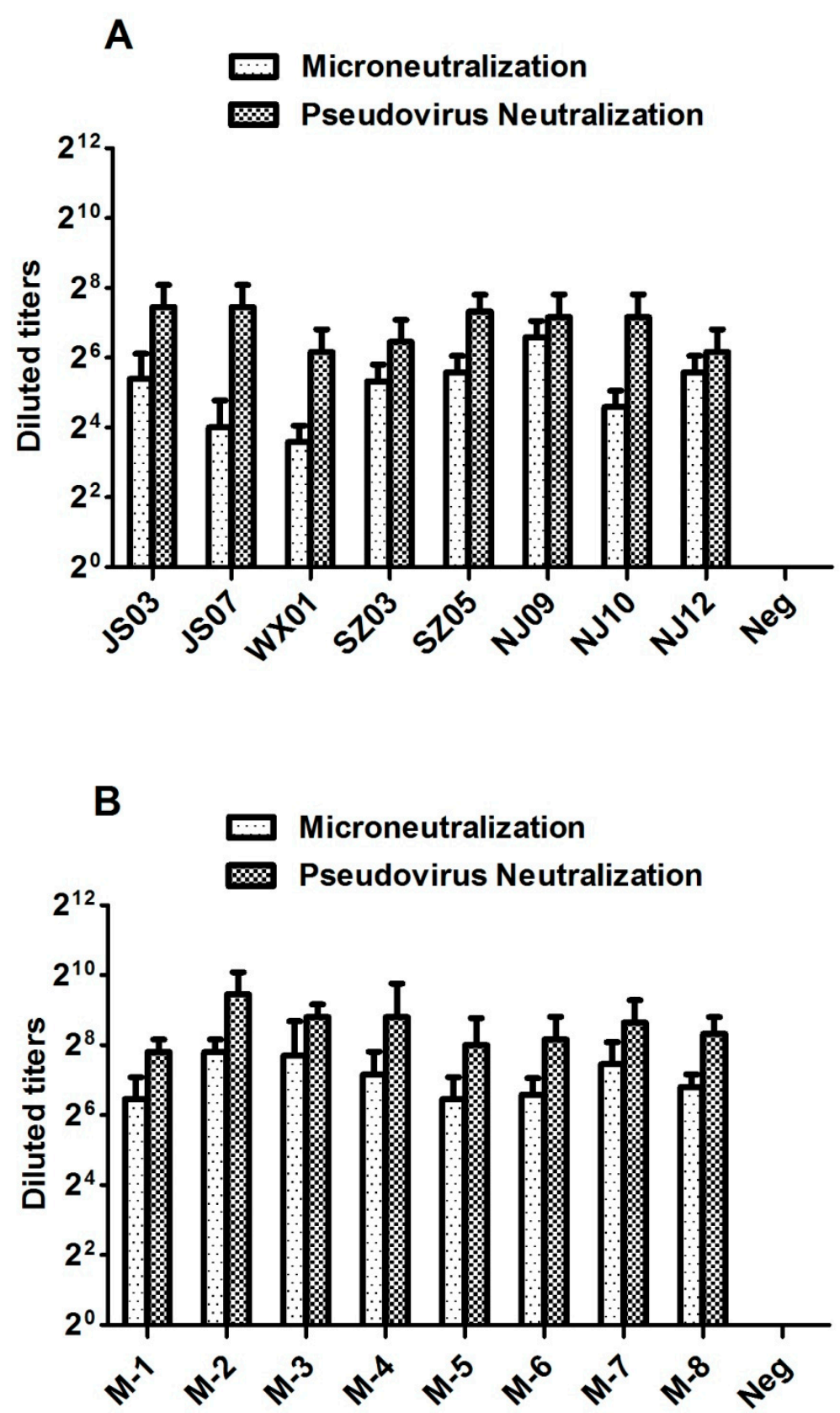

Fig. 4. Compare neutralizing antibody titers between microneutralization and pseudovirus neutralization. Eight human serum samples in convalescent-phase (A) and eight $\mathrm{H} 7 \mathrm{~N} 9$ virus immunized mice serum (B) were all evaluated by microneutralization and pseudovirus neutralization. Data from each group were expressed with a S.D. bar.

\section{References}

1. Gao, R.; Cao, B.; Hu, Y.; Feng, Z.; Wang, D.; Hu, W.; Chen, J.; Jie, Z.; Qiu, H.; Xu, K.; Xu, X.; Lu, H.; Zhu, W.; Gao, Z.; Xiang, N.; Shen, Y.; He, Z.; Gu, Y.; Zhang, Z.; Yang, Y.; Zhao, X.; Zhou, L.; Li, X.; Zou, S.; Zhang, Y.; Li, X.; 
Yang, L.; Guo, J.; Dong, J.; Li, Q.; Dong, L.; Zhu, Y.; Bai, T.; Wang, S.; Hao, P.; Yang, W.; Zhang, Y.; Han, J.; Yu, H.; Li, D.; Gao, G. F.; Wu, G.; Wang, Y.; Yuan, Z.; Shu, Y., Human infection with a novel avian-origin influenza $A$ (H7N9) virus. The New England journal of medicine 2013, 368, (20), 188897.

2. Pushko, P.; Pujanauski, L. M.; Sun, X.; Pearce, M.; Hidajat, R.; Kort, T.; Schwartzman, L. M.; Tretyakova, I.; Chunqing, L.; Taubenberger, J. K.; Tumpey, T. M., Recombinant $\mathrm{H} 7$ hemagglutinin forms subviral particles that protect mice and ferrets from challenge with H7N9 influenza virus. Vaccine 2015, 33, (38), 4975-82.

3. Wilson, J. R.; Guo, Z.; Reber, A.; Kamal, R. P.; Music, N.; Gansebom, S.; Bai, Y.; Levine, M.; Carney, P.; Tzeng, W. P.; Stevens, J.; York, I. A., An influenza A virus (H7N9) anti-neuraminidase monoclonal antibody with prophylactic and therapeutic activity in vivo. Antiviral research 2016, 135, 48-55.

4. Gao, H. N.; Lu, H. Z.; Cao, B.; Du, B.; Shang, H.; Gan, J. H.; Lu, S. H.; Yang, Y. D.; Fang, Q.; Shen, Y. Z.; Xi, X. M.; Gu, Q.; Zhou, X. M.; Qu, H. P.; Yan, Z.; Li, F. M.; Zhao, W.; Gao, Z. C.; Wang, G. F.; Ruan, L. X.; Wang, W. H.; Ye, J.; Cao, H. F.; Li, X. W.; Zhang, W. H.; Fang, X. C.; He, J.; Liang, W. F.; Xie, J.; Zeng, M.; Wu, X. Z.; Li, J.; Xia, Q.; Jin, Z. C.; Chen, Q.; Tang, C.; Zhang, Z. Y.; Hou, B. M.; Feng, Z. X.; Sheng, J. F.; Zhong, N. S.; Li, L. J., Clinical findings in 111 cases of influenza A (H7N9) virus infection. The New England journal of medicine 2013, 368, (24), 2277-85.

5. Alexander, D. J., An overview of the epidemiology of avian influenza. Vaccine 2007, 25, (30), 5637-44.

6. Kageyama, T.; Fujisaki, S.; Takashita, E.; Xu, H.; Yamada, S.; Uchida, Y.; Neumann, G.; Saito, T.; Kawaoka, Y.; Tashiro, M., Genetic analysis of novel avian $\mathrm{A}(\mathrm{H} 7 \mathrm{~N} 9)$ influenza viruses isolated from patients in China, February to April 2013. Euro surveillance : bulletin Europeen sur les maladies transmissibles = European communicable disease bulletin 2013, 18, (15), 20453.

7. Liu, D.; Shi, W.; Shi, Y.; Wang, D.; Xiao, H.; Li, W.; Bi, Y.; Wu, Y.; Li, X.; Yan, J.; Liu, W.; Zhao, G.; Yang, W.; Wang, Y.; Ma, J.; Shu, Y.; Lei, F.; Gao, G. F., Origin and diversity of novel avian influenza A H7N9 viruses causing human infection: phylogenetic, structural, and coalescent analyses. Lancet (London, England) 2013, 381, (9881), 1926-32.

8. Corman, V. M.; Eickmann, M.; Landt, O.; Bleicker, T.; Brunink, S.; EschbachBludau, M.; Matrosovich, M.; Becker, S.; Drosten, C., Specific detection by real-time reverse-transcription PCR assays of a novel avian influenza $A(H 7 N 9)$ strain associated with human spillover infections in China. Euro surveillance: bulletin Europeen sur les maladies transmissibles = European communicable disease bulletin 2013, 18, (16), 20461.

9. Li, Y.; Wu, T.; Qi, X.; Ge, Y.; Guo, X.; Wu, B.; Yu, H.; Zhu, Y.; Shi, Z.; Wang, $\mathrm{H} . ;$ Cui, L.; Zhou, M., Simultaneous detection of hemagglutinin and neuraminidase genes of novel influenza $A(H 7 N 9)$ by duplex real-time reverse transcription polymerase chain reaction. Journal of virological methods 2013, 194, (1-2), 194-6.

10. Wu, J.; Zou, L.; Ni, H.; Pei, L.; Zeng, X.; Liang, L.; Zhong, H.; He, J.; Song, Y.; Kang, M.; Zhang, X.; Lin, J.; Ke, C., Serologic screenings for H7N9 from three sources among high-risk groups in the early stage of H7N9 circulation in Guangdong Province, China. Virology journal 2014, 11, 184. 
11. Zhou, P.; Hong, M.; Merrill, M. M.; He, H.; Sun, L.; Zhang, G., Serological report of influenza A (H7N9) infections among pigs in Southern China. BMC veterinary research 2014, 10, 203.

12. Lin, Y. P.; Yang, Z. F.; Liang, Y.; Li, Z. T.; Bond, H. S.; Chua, H.; Luo, Y. S.; Chen, Y.; Chen, T. T.; Guan, W. D.; Lai, J. C.; Siu, Y. L.; Pan, S. H.; Peiris, J. S.; Cowling, B. J.; PunMok, C. K., Population seroprevalence of antibody to influenza A(H7N9) virus, Guangzhou, China. BMC infectious diseases 2016, $16,(1), 632$.

13. He, F.; Prabakaran, M.; Tan, Y.; Indira, K.; Kumar, S. R.; Kwang, J., Development of dual-function ELISA for effective antigen and antibody detection against $\mathrm{H7}$ avian influenza virus. BMC microbiology 2013, 13, 219.

14. Dong, L.; Bo, H.; Bai, T.; Gao, R.; Dong, J.; Zhang, Y.; Guo, J.; Zou, S.; Zhou, J.; Zhu, Y.; Xin, L.; Li, X.; Xu, C.; Wang, D.; Shu, Y., A combination of serological assays to detect human antibodies to the avian influenza A H7N9 virus. PloS one 2014, 9, (4), e95612.

15. Alvarado-Facundo, E.; Vassell, R.; Schmeisser, F.; Weir, J. P.; Weiss, C. D.; Wang, W., Glycosylation of Residue 141 of Subtype H7 Influenza A Hemagglutinin (HA) Affects HA-Pseudovirus Infectivity and Sensitivity to Site A Neutralizing Antibodies. PloS one 2016, 11, (2), e0149149.

16. Cheresiz, S. V.; Kononova, A. A.; Razumova, Y. V.; Dubich, T. S.; Chepurnov, A. A.; Kushch, A. A.; Davey, R.; Pokrovsky, A. G., A vesicular stomatitis pseudovirus expressing the surface glycoproteins of influenza A virus. Archives of virology 2014, 159, (10), 2651-8.

17. Lu, Y.; Jiang, T., Pseudovirus-based neuraminidase inhibition assays reveal potential H5N1 drug-resistant mutations. Protein \& cell 2013, 4, (5), 356-63.

18. Wu, W.; Li, R.; Li, X.; He, J.; Jiang, S.; Liu, S.; Yang, J., Quercetin as an Antiviral Agent Inhibits Influenza A Virus (IAV) Entry. Viruses 2015, 8, (1).

19. Labrosse, B.; Tourdjman, M.; Porcher, R.; LeGoff, J.; de Lamballerie, X.; Simon, F.; Molina, J. M.; Clavel, F., Detection of extensive cross-neutralization between pandemic and seasonal A/H1N1 Influenza Viruses using a pseudotype neutralization assay. PloS one 2010, 5, (6), e11036.

20. Sapp, M.; Bienkowska-Haba, M., Viral entry mechanisms: human papillomavirus and a long journey from extracellular matrix to the nucleus. The FEBS journal 2009, 276, (24), 7206-16.

21. Wang, Y.; Cui, R.; Li, G.; Gao, Q.; Yuan, S.; Altmeyer, R.; Zou, G., Teicoplanin inhibits Ebola pseudovirus infection in cell culture. Antiviral research 2016, 125, 1-7.

22. Zhao, G.; Du, L.; Ma, C.; Li, Y.; Li, L.; Poon, V. K.; Wang, L.; Yu, F.; Zheng, B. J.; Jiang, S.; Zhou, Y., A safe and convenient pseudovirus-based inhibition assay to detect neutralizing antibodies and screen for viral entry inhibitors against the novel human coronavirus MERS-CoV. Virology journa/2013, 10, 266.

23. Zhang, L.; Lu, J.; Chen, Y.; Shi, F.; Yu, H.; Huang, C.; Cui, L.; Shi, Z.; Jiao, Y.; $\mathrm{Hu}, \mathrm{Y}$., Characterization of Humoral Responses Induced by an H7N9 Influenza Virus-Like Particle Vaccine in BALB/C Mice. Viruses 2015, 7, (8), 4369-84. 